\title{
EDITORIAL
}

\section{No Longer a Distant Dream}

The last decade has seen unprecedented progress in the field of medicine. The advancement in genetics, nanotechnology, biotechnology, development in computer technologies, physics, and other areas has significantly changed or affected the way medicine is being practiced today, particularly in the field of infectious diseases, gene therapy, drug delivery devices, organ transplants, minimally invasive and robotic surgeries, molecular and genetic diagnostics, and bioengineered medical devices.

Human genome has been full decoded and recent advances in sequencing technologies may enhance the role of genetic testing into better patient care. Initially the whole genome sequencing may be limited to newborn screenings for genetic diseases, but later may expand to other settings too providing a large amount of personal genetic data with tremendous impact on the delivery of health services. The pharmaco-genomics is slowly entering in the mainstream medical practice, and helping clinicians in selecting drugs according to each person's own genetic makeup with greater precision in efficacy and safety. In the treatment of cancer there is a gradual shift from cytotoxic drugs to targeted therapeutic drugs, and surgical biopsies for some cancers are routinely subjected to a panel of genetic variants to ascertain if the disease will respond to targeted therapies with better results.

The evidence-based medicine (EBM) has become the focus of health care delivery, and it has extended to virtually every area of medicine. It mandates that clinicians use the best evidence in making decisions while imparting health care to their patients. The patient safety and clinical outcomes have significantly improved due to stringent applications of EBM. A number of guidelines have been promulgated by various professional societies for the prevention and treatment of various diseases, and these are being updated at regular intervals to make the health care services more scientific. These guidelines also have the potential to cut the health care cost wherever feasible.

In the past decade, the medical humanism movement has also emerged as a strong factor in health care sector. It stresses upon the respect for individual patient's values, goals, and preferences with respect to clinical decisions. The patient's right in accepting or rejecting the evidence-based therapy is supreme. The new century health care makes the patient the key component of patient-centered care and informed decision making. Today patients increasingly insist upon their active involvement in the decisions related to their health.

Patient safety and quality of health care goal has now become an important aspect of health care services. Patient-centred care (PCC) is an important measure of health care quality, and the health information technology (IT) plays an important role in achieving this goal. The Institute of Medicine (IOM) defines PCC as care that is respectful of and responsive to individual patient preferences, needs and values. The IOM also stresses upon the importance of these factors in guiding all clinical decisions. The shared decision-making between doctors and patients is central to the patient-centered care, and plays a key role in improving the quality of and safety in healthcare services.

Computers and hand-held devices including smart phones are increasingly playing a role in health care settings. Health IT applications is slowly and steadily making inroads in all aspects of PCC. Health IT has many aspects, such as clinical decisions aids, IT-guided disease management, and telemedicine and telemonitoring systems for the management of a number of diseases, particularly heart ailments, diabetes, asthma, and cancer. Slowly and steadily the positive impact of health IT is becoming apparent 


\section{EDITORIAL}

in the clinical outcomes. Probably a more strong impact of health IT in future will emerge as it further grows and removes the existing lacuna in it.

The importance of health issues in resource-limited countries is also being increasingly recognized, particularly those involving public health and humanitarian challenges, such as research on vaccines and drug development and implementation of public health measures.

Thus, a clinician has to play a number of roles in the present times, and the expectations on him in playing these roles efficiently, is high not only due to increased patient expectations, but also due to increasing medicolegal challenges. In this context, a clinician has to rapidly adapt himself to ever increasing challenges, and impart perfect health care services to all.

\section{Dr. Chandra Prakash Dokwal}

Sr. Consultant \& Coordinator

Department of Respiratory Medicine

Apollo Hospitals Dhaka 